\title{
REALIZABILITY AND NONREALIZABILITY OF DICKSON ALGEBRAS AS COHOMOLOGY RINGS
}

\author{
LARRY SMITH AND R. M. SWITZER
}

\begin{abstract}
Fix a prime $p$ and let $V$ be an $n$-dimensional vector space over $\mathbf{Z} / p$. The general linear group GL $(V)$ of $V$ acts on the polynomial ring $P(V)$ on $V$. The ring of invariants $P(V)^{\mathrm{GL}(V)}$ has been computed by Dickson, and we denote it by $D^{*}(n)$. If we grade $P(V)$ by assigning the elements of $V$ the degree 2, then $D^{*}(n)$ becomes a graded polynomial algebra on generators $Y_{1}, \ldots, Y_{n}$ of degrees $2 p^{n}-$ $2 p^{n-1}, \ldots, 2 p^{n}-2$. The mod $p$ Steenrod algebra acts on $P(V)$ in a unique way compatible with the unstability condition and the Cartan formula. The GL $(V)$ action commutes with the Steenrod algebra action, and so $D^{*}(n)$ inherits the structure of an unstable polynomial algebra over the Steenrod algebra. In this note we determine explicit formulas for the action of the Steenrod algebra on the polynomial generators of $D^{*}(n)$. As a consequence we are able to decide exactly which Dickson algebras can be $\mathbf{Z} / p$ cohomology rings.
\end{abstract}

0. Introduction. Let $p$ denote a prime integer and let $V$ be an $n$-dimensional vector space over the Galois field $\mathbf{Z} / p$. The general linear group $\mathrm{GL}(V)$ of $V$ acts on $P(V)$, the polynomial ring over $V$. The ring of invariants $P(V)^{\mathrm{GL}(V)}$ will be denoted by $D^{*}(n)$. We regard $P(V)$ as being graded by requiring that the elements of $V$ have grading degree 2. Then $D^{*}(n)$ is a graded subalgebra of $P(V)$. The graded polynomial algebra $P(V)$ carries in a unique way the structure of an unstable algebra over the Steenrod algebra (see for example [15,13 or 2] for the definition of the relevant terms) determined by

$$
\begin{aligned}
P^{k} v & =\left\{\begin{array}{ll}
v: & k=0, \\
v^{p}: & k=1, \\
0: & k>1,
\end{array}\right\} \quad \forall v \in V, p>2, \\
\beta v & =0
\end{aligned}
$$

and

$$
S q^{k} v=\left\{\begin{array}{ll}
v: & k=0, \\
v^{2}: & k=2, \\
0: & \text { otherwise, }
\end{array}\right\} \quad p=2 \text { and } \forall v \in V .
$$

The action of GL(V) is compatible with the action of the Steenrod algebra and thus $D^{*}(n)$ inherits the structure of an unstable algebra over the $\bmod p$ Steenrod algebra. The algebra structure of $D^{*}(n)$ has been determined by Dickson [4] (see also (1.5) below) who showed

$$
D^{*}(n) \simeq \mathbf{Z} / p\left[Y_{1}, \ldots, Y_{n}\right]: \quad \operatorname{deg} Y_{i}=2 p^{n}-2 p^{n-i} ; \quad p \text { odd } ; i=1, \ldots, n .
$$

Received by the editors June 28, 1982.

1980 Mathematics Subject Classification. Primary 55F40; Secondary 55F35. 
The Dickson algebra plays a central role in determining which algebras over the Steenrod algebra occur as rings of invariants (see e.g. [2] where this is well disguised or [14] where it is brought to the fore). For large primes this in turn shows that the polynomial algebras that occur as cohomology rings are exactly those constructed in [3]. It is therefore quite natural to ask if the Dickson algebra itself can occur as a cohomology ring. Our main result settles this question for all primes. Specifically we show

THEOREM. Let $p$ be a prime and $n$ a positive integer. Then there exists a topological space $X(n)$ such that

$$
H^{*}(X(n) ; \mathbf{Z} / p) \simeq D^{*}(n)
$$

as algebras over the Steenrod algebra iff $n=1$, or $n=2$ and $p \leqslant 3$.

For $n=1, D^{*}(1) \simeq \mathbf{Z} / p[Y]$ where $\operatorname{deg} Y=2 p-2$, and a relevant space $X(1)$ realizing $D^{*}(1)$ is a delooping of $S^{2 p-3}(p)$ which has been constructed by Holzager [8] and Sullivan [17]. A space with cohomology mod 3 isomorphic to $D^{*}(2) \simeq$ $\mathrm{Z} / 3\left[Y_{1}, Y_{2}\right], \operatorname{deg} Y_{1}=12, \operatorname{deg} Y_{2}=16$, has recently been constructed by Zabrodsky [18], utilizing Friedlander's exceptional isogeny [6] $\theta: B F_{4}\left(\frac{1}{2}\right) \rightarrow B F_{4}\left(\frac{1}{2}\right)$. This corrects an oversight in [13] that was noted in [5]. (In [5] however the type $(8,12)$ mod 3 is incorrectly eliminated as a cohomology ring. The type in question occurs as for example the ring of invariants $P(V)^{\mathrm{SL}(V)}$ where $V$ is a 2-dimensional vector space over $\mathbf{Z} / 3$. However more detailed calculation shows this latter type is not a cohomology ring.) For $p=2, D^{*}(1)$ and $D^{*}(2)$ are the $\mathbf{Z} / 2$ cohomology of $C P(\infty)$ and $B S U(3)$ respectively.

The organization of this paper is as follows. In $\S 1$ we determine the structure of $D^{*}(n)$ as an unstable algebra over the Steenrod algebra. In the process we rederive the theorem of Dickson in the spirit of Adams and Wilkerson [2, §§3 and 5]. Combining this with elementary computations with secondary operations we arrive at the nonrealization theorem in $\S 2$. In $\$ 3$ we discuss the (really odd !) prime 2.

For further connections between the Dickson algebra and algebraic topology we refer the reader to [15]. We wish to thank Clarence Wilkerson for a useful correspondence concerning the case $p=2$.

1. The Steenrod algebra structure of the Dickson algebra. To simplify the exposition in this section we will suppose that $p$ is an odd prime. The modifications for the prime 2 are left to the reader. The results are at any rate formulated in $\S 2$.

NOTATIONS AND DEFINITIONS. We denote by $\rho^{\rho *}$ the algebra of Steenrod reduced powers (no Bockstein). The dual Hopf algebra $\mathscr{P}_{*}$ has been determined by Milnor [11] who showed

$$
\mathscr{P}_{*} \simeq \mathbf{Z} / p\left[\xi_{1}, \ldots, \xi_{n}, \ldots\right]: \quad \operatorname{deg} \xi_{i}=2 p^{i}-2 ; \quad i>0 .
$$

Thus a basis for the primitive elements of $\mathcal{P} *$ is given by the elements $P^{\Delta_{1}} \in \mathscr{P}^{2 p^{t}-2}$ dual to $\xi_{i}$ in the usual monomial basis of $\mathscr{P}_{*}$. The elements $P^{\Delta_{1}}$ can be inductively defined by

$$
P^{\Delta_{1}}:=P^{1}, \quad P^{\Delta_{i+1}}:=\left[P^{p^{i}}, P^{\Delta_{i}}\right]
$$


By an unstable algebra $A^{*}$ over the Steenrod algebra we understand an evenly graded algebra over the Hopf algebra $\$ p^{*}$ in the usual sense [12] that satisfies in addition the unstability conditions

$$
P^{k}(a)= \begin{cases}a^{p}: & 2 k=\operatorname{deg} a, \\ 0: & 2 k>\operatorname{deg} a .\end{cases}
$$

The category of unstable algebras over the Steenrod algebra is denoted by UnAl/P?*.

Being primitive, the elements $P^{\Delta_{1}}$ act as derivations on any $A^{*} \in U n A l / \rho^{*} *$. It is convenient to introduce a further derivation $P^{\Delta^{0}}$ of degree zero by the rule

$$
P^{\Delta_{0}}(a)=d a: \quad \forall a \in A^{2 d} .
$$

(N.B. $P^{\Delta_{0}} \notin$.?*) The following result of Adams and Wilkerson [2, Theorem 5.1] is fundamental.

Proposition 1.1. Let $A^{*} \in U n A l /$ P. $^{*}$ and suppose that there is an upper bound on the number of algebraically independent elements in $A^{*}$. Then there exists an integer $n \geqslant 0$ such that any $n+1$ derivations $P^{\Delta_{r_{0}}}, \ldots, P^{\Delta_{r_{n}}}$ are linearly dependent, but any $n$ distinct derivations $P^{\Delta}$, are linearly independent.

Linear dependence and independence as used in (1.1) should be understood as referring to the left $A^{*}$ module structure of $\operatorname{End}\left(A^{*}\right)$. An essential point in the proof of (1.1) is the following derivation lemma, which we will presently need ourselves.

Derivation lemma. Let $k$ be a perfect field and $A^{*}$ a graded $k$ algebra. Suppose we are given derivations

$$
\partial_{1}, \ldots, \partial_{n}: A^{*} \rightarrow A^{*},
$$

and elements $x_{1}, \ldots, x_{n} \in A^{*}$ such that

$$
\operatorname{Det}\left(\partial_{i} x_{j}\right) \neq 0
$$

Then $x_{1}, \ldots, x_{n} \in A^{*}$ are algebraically independent.

For a proof see [19, pp. 126-127].

Definition. Let $V$ be an $n$-dimensional vector space over $\mathbf{Z} / p$. Set $S^{*}:=P(V)$ and introduce the polynomial

$$
f(X):=\prod_{i \in V}(X-v) \in S^{*}[X]
$$

where $X$ is an indeterminant of degree 2 .

Note that the polynomial $f(X)$ is invariant under the action of $\operatorname{GL}(V)$ on $S^{*}[X]$, so the coefficients belong to $D^{*}(n)$, so there are elements $Y_{1}, \ldots, Y_{n} \in D^{*}(n)$ such that

$$
f(X)=X^{p^{n}}+Y_{1} X^{p^{n-1}}+\cdots+Y_{n} X .
$$

In addition the roots of $f(X)$ form the vector space $V$.

The derivation lemma shows that the derivations $P^{\Delta_{0}}, \ldots, P^{\Delta_{n}}$ are linearly dependent over $S^{*}$. Thus we may choose $d_{0}, \ldots, d_{n} \in S^{*}$ such that

$$
d_{n} P^{\Delta_{0}}+\cdots+d_{0} P^{\Delta_{n}}=0 \in \operatorname{End}\left(S^{*}\right) .
$$


Having made such a choice introduce the polynomial

$$
\Delta(X):=d_{n} X+d_{n-1} X^{p}+\cdots+d_{0} X^{p^{n}} \in S^{*}[X] .
$$

LEMMA 1.2. With the notations preceding $\Delta(X)=d_{0} f(X)$.

Proof. The action of the derivations $P^{\Delta}$, on 2-dimensional classes can be computed inductively from the instability condition and the formula $P^{\Delta_{i+1}}=$ $\left[P^{p^{\prime}}, P^{\Delta_{1}}\right]$. The result is

$$
P^{\Delta_{i}} v=v^{p^{\prime}}: \quad i=0,1, \ldots, \operatorname{deg} v=2 .
$$

Thus for $v \in V$ we have

$$
0=\left(d_{n} P^{\Delta_{0}}+\cdots+d_{0} P^{\Delta_{n}}\right)(v)=d_{n} v+d_{n-1} v^{p}+\cdots+d_{0} v^{p^{n}}=\Delta(v) .
$$

That is the elements of $V$ are roots of the polynomial $\Delta(X)$. From the definition of $f(X)$ it follows that $f(X)$ divides $\Delta(X)$ in $S^{*}[X]$. But $\Delta(X)$ and $f(X)$ both have degree $p^{n}$ in $X$, so $\Delta(X)=d_{0} f(X)$ as claimed.

LEMma 1.3. Suppose $A^{*} \in U n A l /{ }^{\rho *}$ and

$$
h_{0} P^{\Delta_{0}}=h_{1} P^{\Delta_{r_{1}}}+\cdots+h_{n} P^{\Delta_{r_{n}}} \in \operatorname{End}(A),
$$

where $h_{0}$ is a pth power. Then

$$
h_{0} P^{\Delta_{r}}=\left(P^{\Delta_{r}} h_{1}\right) P^{\Delta_{r_{1}}}+\cdots+\left(P^{\Delta_{r}} h_{n}\right) P^{\Delta_{r_{n}}}
$$

for all $r \geqslant 0$.

Proof. First of all recall the commutation relation

$$
\left[P^{\Delta_{1}}, P^{\Delta_{1}}\right]= \begin{cases}0: & j \neq 0 \\ P^{\Delta_{i}:} & j=0 .\end{cases}
$$

Let $x \in A^{*}$ be arbitrary. From $(*)$ we get

$$
h_{0} P^{\Delta_{0}}(x)=h_{1} P^{\Delta_{r_{1}}}(x)+\cdots+h_{n} P^{\Delta_{r_{n}}}(x) \text {. }
$$

Apply $P^{\Delta_{r}}$ to both sides of $(* *)$ and note that $P^{\Delta_{r}} h_{0}=0$ since $h_{0}$ is a $p$ th power, to obtain for $r>0$

$$
\begin{aligned}
\left(P^{\left.\Delta_{r} * x\right)} \quad h_{0} P^{\Delta_{r}} P^{\Delta_{0}}(x)=\right. & P^{\Delta_{r}}\left(h_{1}\right) P^{\Delta_{r_{1}}}(x)+\cdots+P^{\Delta_{r}}\left(h_{n}\right) P^{\Delta_{r_{n}}}(x) \\
& +h_{1} P^{\Delta_{r}} P^{\Delta_{r_{1}}}(x)+\cdots+h_{n} P^{\Delta_{r}} P^{\Delta_{r_{n}}}(x) \\
= & \left(P^{\Delta_{r}} h_{1}\right) P^{\Delta_{r_{1}}}(x)+\cdots+P^{\Delta_{r}}\left(h_{n}\right) P^{\Delta_{r_{n}}(x)} \\
& +h_{1} P^{\Delta_{r_{1}}} P^{\Delta_{r}}(x)+\cdots+h_{n} P^{\Delta_{r_{n}}} P^{\Delta_{r}}(x) \\
= & \left(\left(P^{\Delta_{r}} h_{1}\right) P^{\Delta_{r_{1}}}+\cdots+\left(P^{\Delta_{r}} h_{n}\right) P^{\Delta_{r_{n}}}\right)(x) \\
& +\left(h_{1} P^{\Delta_{r_{1}}}+\cdots+h_{n} P^{\Delta_{r_{n}}}\right)\left(P^{\Delta_{r}}(x)\right) \\
\text { by }(*) \text { applied }= & \left(\left(P^{\Delta_{r}} h_{1}\right) P^{\Delta_{r_{1}}}+\cdots+\left(P^{\Delta_{r}} h_{n}\right) P^{\Delta_{r_{n}}}\right)(x) \\
\text { to } P^{\Delta_{r}}(x) \quad & +h_{0} P^{\Delta_{0}} P^{\Delta_{r}}(x),
\end{aligned}
$$


so rearranging gives

$$
h_{0} P^{\Delta_{r}}(x)=h_{0}\left[P^{\Delta_{r}}, P^{\Delta_{0}}\right](x)=\left(\left(P^{\Delta_{r}} h_{1}\right) P^{\Delta_{r_{1}}}+\cdots+\left(P^{\Delta_{r}} h_{n}\right) P^{\Delta_{r_{n}}}\right)(x)
$$

which yields the required result since $x$ is arbitrary.

The following lemma is the result of the relaxing effect of Appenzellerland on one of the authors.

LEMMA 1.4. Suppose $A^{*} \in U n A l / \rho *$ contains at most finitely many algebraically independent elements. Let $n$ be as in (1.1) and choose $d_{0}, \ldots, d_{n} \in A^{*}$, with $d_{n}$ a pth power, such that $d_{n} P^{\Delta_{0}}+\cdots+d_{0} P^{\Delta_{n}}=0 \in \operatorname{End}\left(A^{*}\right)$. Then

$$
P^{\Delta_{i}} d_{j}=\left\{\begin{array}{cl}
-d_{n}: & i+j=n, 0 \leqslant j \leqslant n, \\
0: & \text { otherwise, } 1 \leqslant i \leqslant n .
\end{array}\right.
$$

(N.B. By (1.1) none of the coefficients $d_{0}, \ldots, d_{n}$ can vanish.)

Proof. From (1.3) we get

$$
-d_{n} P^{\Delta_{i}}=\left(P^{\Delta_{i}} d_{n-1}\right) P^{\Delta_{1}}+\cdots+\left(P^{\Delta_{i}} d_{0}\right) P^{\Delta_{n}} .
$$

Rearranging then gives

$$
0=\left(P^{\Delta_{i}} d_{n-1}\right) P^{\Delta_{1}}+\cdots+\left(P^{\Delta_{i}} d_{n-i}+d_{n}\right) P^{\Delta_{i}}+\cdots+\left(P^{\Delta_{i}} d_{0}\right) P^{\Delta_{n}} .
$$

But $P^{\Delta_{1}}, \ldots, P^{\Delta_{n}}$ are linearly independent by hypothesis, so

$$
P^{\Delta_{i}} d_{n-j}=\left\{\begin{array}{cl}
0: & i \neq j, \\
-d_{n}: & i=j,
\end{array}\right.
$$

as required.

THEOREM 1.5 (DICKSON). $D^{*}(n) \simeq \mathbf{Z} / p\left[Y_{1}, \ldots, Y_{n}\right]$ where $\operatorname{deg} Y_{i}=2 p^{n}-2 p^{n-i}$ and moreover

$$
X^{p^{n}}+Y_{1} X^{p^{n-1}}+\cdots+Y_{n} X=\prod_{v \in V}(X-v)
$$

in $S^{*}[X]$

Proof. First of all note that $P^{\Delta_{1}}, \ldots, P^{\Delta_{n}}$ are linearly independent on $S^{*}$. To see this, choose a basis $v_{1}, \ldots, v_{n}$ for $V$ and consider

$$
\operatorname{Det}\left(P^{\Delta_{i}} v_{j}\right)=\left[\begin{array}{lr}
v_{1}^{p}, \ldots, v_{n}^{p} \\
\vdots & \\
v_{1}^{p^{n}}, \ldots, v_{n}^{p^{n}}
\end{array}\right] .
$$

Note that upon expanding the coefficient of the monomial $v p v p^{2} \cdots v_{n}^{p^{n}}$ is 1 , so $\operatorname{Det}\left(P^{\Delta_{i}} v_{j}\right) \neq 0$, and therefore the integer of Proposition 1.1 is in fact $n$, the 
dimension of $V$. So from Lemmas (1.2) and (1.4) we find

$$
\begin{aligned}
& P^{\Delta_{1}}\left(Y_{k}\right)=P^{\Delta_{i}} \frac{d_{k}}{d_{0}}=\frac{d_{0} P^{\Delta_{i}} d_{k}-d_{k} P^{\Delta_{1}} d_{0}}{d_{0}^{2}} \\
& = \begin{cases}\frac{-d_{0} d_{n}}{d_{0}^{2}}=\frac{-d_{n}}{d_{0}}=-Y_{n}: & i=n-k ; k>0, \\
\frac{d_{k} d_{n}}{d_{0}^{2}}=Y_{k} Y_{n}: & i=n, \\
0: & \text { otherwise, }\end{cases}
\end{aligned}
$$

and thus

$$
P^{\Delta} Y_{k}=\left\{\begin{array}{cl}
-Y_{n}: & i=n-k \\
Y_{k} Y_{n}: & i=n, \\
0: & \text { otherwise. }
\end{array}\right.
$$

Therefore

$$
\operatorname{Det}\left(P^{\Delta_{1}} Y_{k}\right)=\operatorname{Det}\left[\begin{array}{cccc}
0 & \cdots & -Y_{n} & 0 \\
-Y_{n} & . \cdot & & \vdots \\
Y_{1} Y_{n} & \cdots & Y_{n-1} Y_{n} & Y_{n} Y_{n}
\end{array}\right]=(-1)^{n-1} Y_{n}^{n+1} \neq 0
$$

Therefore by the derivation lemma $Y_{1}, \ldots, Y_{n} \in S^{*}$ are algebraically independent.

Let $H^{*}:=\mathbf{Z} / p\left[Y_{1}, \ldots, Y_{n}\right]<D^{*}(n)<S^{*}$. Let $F()$ be the field of fractions functor. Note that $F\left(S^{*}\right)$ is the field of rational functions on $V$, and that the elements of $V$ all satisfy the equation $f(X)=0$ where

$$
f(X)=\prod_{v \in V}(X-v)=Y_{n} X+\cdots+X^{p^{n}} \in H^{*}[X] .
$$

Thus we conclude that $F\left(S^{*}\right)$ is in fact the splitting field of the polynomial $f(X)$ over $F\left(H^{*}\right)$. Since the polynomial $f(X)$ defines an additive function upon evaluating at elements of degree 2, it follows that the Galois group of $F\left(S^{*}\right)$ over $F\left(H^{*}\right)$ is a subgroup of $\mathrm{GL}(V)$. (N.B. The extension is Galois since $f(X)$ is separable.) Thus $D^{*}(n) \leqslant S^{*} \cap F\left(H^{*}\right)$. Since $S^{*}$ is integral over $H^{*}$ and $H^{*}$ is integrally closed (in its field of fractions) it follows that $S \cap F\left(H^{*}\right)=H^{*}$. Thus $D^{*}(n) \leqslant H^{*} \leqslant D^{*}(n)$ and the result follows.

Corollary to the Proof of (1.5). In the Dickson algebra $D^{*}(n)$ we have

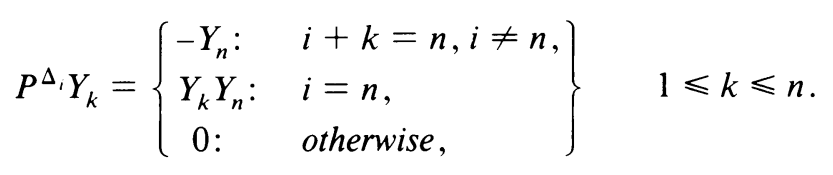

CorollaRY 1.6. In the Dickson algebra $D^{*}(n)$ we have

$$
P^{p^{j}} Y_{k}= \begin{cases}Y_{k+1}: & \text { if } j+k=n-1, \\ -Y_{1} Y_{k}: & \text { if } j=n-1, k>1, \\ 0: & \text { otherwise. }\end{cases}
$$


In particular the action of the Steenrod algebra on the module of indecomposables $Q D^{*}(n)$ is given by the following scheme.

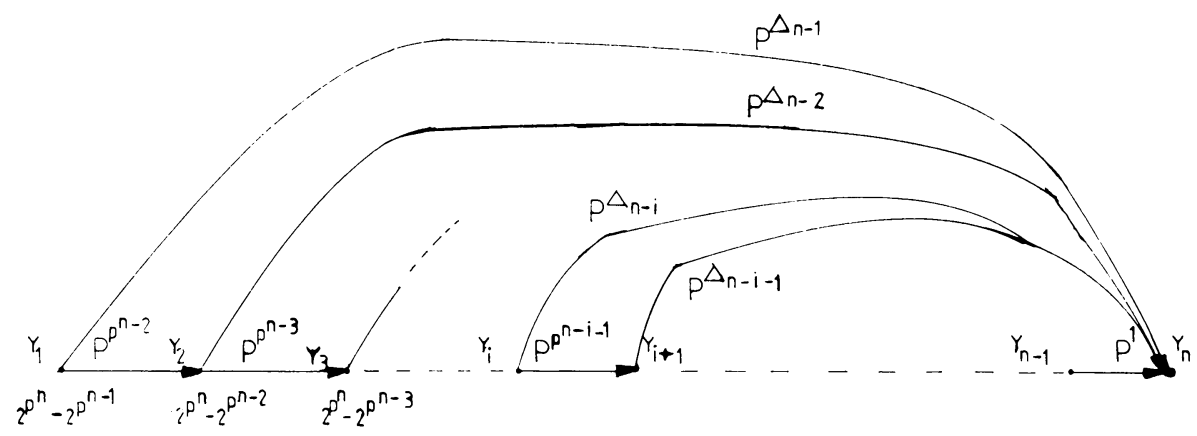

ProOF. Note that as

$$
\operatorname{deg} Y_{i}=2 p^{n}-2 p^{n-i},
$$

the degree of $Y_{i}$ increases with $i$. So $Y_{1}$ has the lowest degree and $Y_{n}$ the largest. Note further that

$$
\operatorname{deg} Y_{1}^{2}-\operatorname{deg} Y_{n}=4 p^{n}-4 p^{n-1}-2 p^{n}+2=2 p^{n-1}(p-2)+2>0 .
$$

Thus in the degrees $2 p^{n}-2 p^{n-i}$ there are no decomposable elements, and hence

$$
P^{p^{n-1-1}} Y_{i}=a Y_{i+1}: \quad a \in \mathbf{Z} / p .
$$

To compute $a$, we apply the corollary to (1.5) to get

$$
P^{\Delta_{n-1},} P^{p^{n-1}} Y_{i}=-a Y_{n} \text { and } P^{\Delta_{n-1-1}} Y_{i}=0 .
$$

So by the commutation rule and the corollary to (1.5) again we get

$$
-Y_{n}=P^{\Delta_{n-1}} Y_{i}=\left[P^{\Delta_{n-\imath-1}}, P^{p^{n-1-1}}\right]\left(Y_{i}\right)=-a Y_{n}
$$

giving $a=1$, that is

$$
P^{p^{n-1-1}} Y_{i}=Y_{i+1} \text {. }
$$

Next consider the case $P^{p^{n-1}} Y_{k}$. For degree reasons we must have

$$
P^{p^{n-1}} Y_{k}=a Y_{1} Y_{k} ; \quad a \in \mathbf{Z} / p \text {. }
$$

To see this, note that

$$
\begin{aligned}
\operatorname{deg} P^{p^{n-1}} Y_{k} & =2 p^{n-1}(p-1)+2 p^{n}-2 p^{n-k}=2 p^{n}-2 p^{n-1}+2 p^{n}-2 p^{n-k} \\
& =\operatorname{deg} Y_{1}+\operatorname{deg} Y_{k},
\end{aligned}
$$

and $D(n)^{*}$ in this degree is one dimensional over $\mathbf{Z} / p$. To compute $a \in \mathbf{Z} / p$ we apply $P^{\Delta_{n-1}}$ to (*) giving by the corollary to (1.5):

$$
\begin{aligned}
\left(P^{\left.\Delta_{n-1}(*)\right)} \quad P^{\Delta_{n-1}} P^{p^{n-1}} Y_{k}\right. & =a\left[\left(P^{\Delta_{n-1}} Y_{1} Y_{k}+Y_{1}\left(P^{\Delta_{n-1}} Y_{k}\right)\right]\right. \\
& =a\left[-Y_{n} Y_{k}-\delta_{1, k} Y_{k} Y_{n}\right] \\
& =-a\left(1+\delta_{1, k}\right) Y_{k} Y_{n} .
\end{aligned}
$$


On the other hand

$$
P^{\Delta_{n-1} Y_{k}}=\left\{\begin{array}{cl}
0: & k \neq 1 \\
-Y_{n}: & k=1 .
\end{array}\right.
$$

Therefore for $k \neq 1$ we have

$$
Y_{k} Y_{n}=P^{\Delta_{n}} Y_{k}=\left[P^{\Delta_{n-1}}, P^{p^{n-1}}\right]\left(Y_{k}\right)=-a Y_{k} Y_{n}+0=-a Y_{k} Y_{n}
$$

and therefore $a=-1$, that is

$$
P^{p^{n-1}} Y_{k}=-Y_{1} Y_{k}: \quad k \neq 1 .
$$

Finally for $k=1$ we get by applying the corollary to (1.5), $P^{\Delta_{n-1}(*)}$ for $k=1$, and the preceding for $k=n$, that

$$
\begin{aligned}
Y_{1} Y_{n} & =P^{\Delta_{n}} Y_{1}=\left[P^{p^{n-1}}, P^{\Delta_{n-1}}\right]\left(Y_{1}\right) \\
& =(2 a) Y_{1} Y_{n}-P^{p^{n-1}} Y_{n}=(1+2 a) Y_{1} Y_{n}
\end{aligned}
$$

so $a=0$ and $P^{p^{n-1}} Y_{1}=0$ as required.

Summarizing we have shown

$$
P^{p^{j}}\left(Y_{k}\right)=\left\{\begin{array}{cl}
Y_{k+1}: & \text { if } j=n-k-1, \\
-Y_{1} Y_{k}: & \text { if } j=n-1, k>1, \\
0: & \text { if } j=n-1, k=1,
\end{array}\right.
$$

and so it remains to consider $P^{p^{\prime}}\left(Y_{k}\right)$ for $j \neq n-1, n-k-1$. First of all note that

$$
\operatorname{deg} Y_{k}=2 p^{n}-2 p^{n-k}<2 p^{n}
$$

so

$$
P^{p^{j}} Y_{k}=0: \quad j \geqslant n \text {. }
$$

Thus we need only consider values of $j \leqslant n-2$. Next note, under the assumption $j \leqslant n-2$ that

$$
\operatorname{deg} P^{p^{j}} Y_{k}<\operatorname{deg} P^{p^{n-1}} Y_{n}=2 p^{n-1}(p-1)+2 p^{n}-2=\operatorname{deg}\left(Y_{1} Y_{n}\right) .
$$

Finally note that through dimension $2 p^{n-1}(p-1)+2 p^{n}-2$ inclusive an additive basis for $D^{*}(n)$ is given by the monomials

$$
Y_{1}, \ldots, Y_{n}, Y_{1}^{2}, Y_{1} Y_{2}, \ldots, Y_{1} Y_{n},
$$

as a little bookkeeping shows. Further degree calculations give (again for $j \leqslant n-1$ )

$$
\begin{gathered}
\operatorname{deg} P^{p^{j}} Y_{i}=\left\{\begin{array}{l}
2(p-1)\left[p^{n-1}+\cdots+2 p^{j}+\cdots+p^{n-i}\right]: \quad j \geqslant n-i, \\
2(p-1)\left[p^{n-1}+\cdots+p^{n-i}+p^{j}\right]: \quad j<n-i,
\end{array}\right. \\
\operatorname{deg} Y_{r}=2(p-1)\left[p^{n-1}+\cdots+p^{n-r}\right], \\
\operatorname{deg} Y_{1} Y_{s}=2(p-1)\left[2 p^{n-1}+p^{n-2}+\cdots+p^{n-s}\right] .
\end{gathered}
$$

Thus the uniqueness of the $p$-adic exparsion shows that $P^{p^{j}} Y_{i}$ for $i \leqslant n-1$ lands in a nonzero degree iff $j=n-i-1$ (when $r=i+1$ ) or $j=n-1$ (when $s=i$ ) as was to be shown. 
REMARK. It is not hard to show that Corollary 1.6 characterizes $D^{*}(n)$ as an unstable algebra over the Steenrod algebra (see [15]).

2. Nonrealization results. With the explicit formulas of the preceding section for the action of $P^{p^{k}}$ on the generators of the Dickson algebra, it is now an easy matter to prove

THEOREM 2.1. Let $p$ be a prime and $n$ a positive integer. If there exists a topological space $X$ such that $H^{*}(X ; \mathbf{Z} / p) \simeq D^{*}(n)$, then either $n=1$ or $n=2$ and $p \leqslant 3$.

Proof. Consider first the case of $p$ odd. If $n>2$ from (1.6) we see $P^{p} Y_{n-2}=Y_{n-1}$. Assume that there exists a space $X$ such that $H^{*}(X ; \mathbf{Z} / p) \simeq D^{*}(n)$. Then we can apply [10] to write

$$
Y_{n-1}=P^{p} Y_{n-2}=\beta \Lambda Y_{n-2}+P^{p-2} \Re Y_{n-2}
$$

where $\Lambda$ and $\mathscr{R}$ are certain secondary operations of degrees $2 p(p-1)-1$ and $4(p-1)$ respectively. For degree reasons one sees that

$$
\Lambda Y_{n-2}=0=\Re Y_{n-2},
$$

which leads to the contradiction that $Y_{n-1}=0$. For $n=2$ we use the formulae

$$
P^{p} Y_{2}=-Y_{1} Y_{2}, \quad P^{1} Y_{2}=0
$$

obtained from (1.6). Assuming $H^{*}(X ; \mathbf{Z} / p) \simeq D^{*}(2)$ we conclude (since $\beta=0$ on $\left.D^{*}(2)\right)$

$$
-Y_{1} Y_{2}=P^{p} Y_{2}=P^{p-2} \Re Y_{2}
$$

and hence $6 R Y_{2} \neq 0$. Note

$$
\operatorname{deg} Y_{2} \leqslant \operatorname{deg} \Re Y_{2}<\operatorname{deg} Y_{1} Y_{2},
$$

so the only chance for $\mathcal{R} Y_{2}$ to be nonzero is

$$
\Re Y_{2}=b Y_{1}^{2}: \quad b \neq 0 \in \mathbf{Z} / p
$$

which gives upon taking degrees

$$
4(p-1)+2 p^{2}-2=2\left(2 p^{2}-2 p\right),
$$

whose only solution is $p=3$.

For $p=2$ note that the Steenrod algebra action is given by

$$
\mathrm{Sq}^{2^{i+1}} Y_{j}= \begin{cases}Y_{j+1}: & i+j=n-1, \\ Y_{1} Y_{n}: & j=n-1, \\ 0: & \text { otherwise. }\end{cases}
$$

So for $n \geqslant 5$ we find,

$$
\mathrm{Sq}^{16} Y_{n-4}=Y_{n-3}
$$

whereas

$$
\mathrm{Sq}^{2^{i}} Y_{n-4}=0, \quad 1=1,2,3
$$


Applying the decomposition formula for $\mathrm{Sq}^{16}$ and reasoning as before eliminates $D^{*}(n)$ for $n \geqslant 5$ as a cohomology ring. The cases $n=3$ and 4 are eliminated by [7, 1.2].

Combining (2.1) with $[7,18$, p.73 and 10,4.3] we arrive at the following result.

THEOREM 2.2. A necessary and sufficient condition that $D^{*}(n)$ be the $\mathbf{Z} / p$ cohomology of a space is that either $n=1$, or $n=2$ and $p \leqslant 3$.

3. The (odd) prime 2. For $p=2$ the Steenrod algebra acts unstably on the graded polynomial algebra $P(W)$, where the generators $w \in W$ all have degree 1 , the action being given by

$$
\mathrm{Sq}^{i}(w)= \begin{cases}w: & i=0 \\ w^{2}: & i=1, \\ 0: & i>1 .\end{cases}
$$

The algebra $\tilde{D}^{*}(n):=P(W)^{\mathrm{GL}(W)}$ is then an unstable algebra over the Steenrod algebra. The structure of these Dickson algebras is given by the following result, analogous to those of $\S 1$.

THEOREM 3.1. $\tilde{D}^{*}(n) \simeq \mathbf{Z} / 2\left[Z_{1}, \ldots, Z_{n}\right]$ where $\operatorname{deg} Z_{i}=2^{n}-2^{n-i}$. The action of the Steenrod algebra is given by

$$
\mathrm{Sq}^{\Delta_{1}} Z_{j}= \begin{cases}Z_{n}: & i+j=n, \\ 0: & \text { otherwise, }\end{cases}
$$

where $\mathrm{Sq}^{\Delta_{i}} \in \mathbb{Q}^{2^{\prime}-1}(2)$ is the primitive element ( usually denoted $Q_{i}$ ) and

$$
\mathrm{Sq}^{2} Z_{j}= \begin{cases}Z_{j+1}: & i+j=n-1, \\ Z_{1} Z_{j}: & i=n-1, \\ 0: & \text { otherwise. }\end{cases}
$$

Corollary 3.2. For $n \geqslant 6, \tilde{D}^{*}(n)$ is not the $\mathbf{Z} / 2$ cohomology of any space.

Proof. For $n \geqslant 6$ one has

$$
\mathrm{Sq}^{16} Z_{n-5}=Z_{n-4}
$$

whereas

$$
\mathrm{Sq}^{2} Z_{n-5}=0: \quad i=1,2,3
$$

and therefore as in the case of $D^{*}(n)$ one sees that $\tilde{D}^{*}(n)$ cannot be a cohomology ring.

The algebras $\tilde{D}^{*}(1), \tilde{D}^{*}(2)$ and $\tilde{D}^{*}(3)$ occur as the $\mathbf{Z} / 2$ cohomology rings of the spaces $\mathbf{R} P(\infty), B S O(3)$ and $B G_{2}$ respectively. The remaining two cases, $\tilde{D}^{*}(4)$ and $\tilde{D}^{*}(5)$, have type $(8,12,14,15)$ and $(16,24,28,30,31)$, and it is an unsettled problem if they can occur as cohomology rings.

\section{REFERENCES}

1. J. F. Adams, The non-existence of elements of Hopf invariant one, Ann. of Math. (2) 72 (1960), $20-104$.

2. J. F. Adams and C. W. Wilkerson, Finite H-spaces and algebras over the Steenrod algebra, Ann. of Math. (2) 111 (1980), 95-143. 
3. A. Clark and J. Ewing. The realization of polynomial algebras as cohomologv rings. Pacific J. Math. 50 (1974), 425-434.

4. L. E. Dickson, A fundamental sistem of invariants of the general modular linear group with a solution of the form problem. Trans. Amer. Math. Soc. 12 (1911), 75-98.

5. J. Ewing, Sphere hundles over spheres as loop spaces mod p. Duke Math. J. 40 (1973), 157-162.

6. E. M. Friedlander, Exceptional isogenies and the classifying spaces of simple Lie groups, Ann. of Math. (2) 101 (1975), 510-520.

7. J. R. Hubbuck, Generalized cohomologv operations and H-spaces of low rank, Trans. Amer. Math. Soc. 141 (1969), 335-360.

8. R. Holzsager, $H$-spaces of category $\leqslant 2$, Topology 9 (1970), $211-216$.

9. S. Lang, Algebra, Addison-Wesley, Reading, Mass., 1965.

10. A. Liulevicius, The factorization of cyclic reduced powers by secondary cohomology operations, Mem. Amer. Math. Soc. no. 42 (1962).

11. J. Milnor, The Steenrod algebra and its dual, Ann. of Math. (2) 67 (1958), 150-171.

12. J. Milnor and J. C. Moore, The structure of Hopf algebras, Ann. of Math. (2) 81 (1965), 211-264.

13. R. Nakagawa and S. Ochiai, On the dimension of generators of a polynomial algebra over the Mod $p$ Steenrod algehra, Proc. Japan Acad. Ser. A Math. Sci. 43 (1967), 932-936.

14. L. Smith, On the type of an associative H-space of rank 2, Tôhoku Math. J. 20 (1968), 511-515.

15. L. Smith and R. M. Switzer, Variations on a theme of Adams and Wilkerson (to appear).

16. N. E. Steenrod and D. B. A. Epstein, Cohomology operations, Ann. of Math. Studies, no. 50, Princeton Univ. Press, Princeton, N. J., 1962.

17. D. Sullivan, Genetics of homotopy theory and the Adams conjecture, Ann. of Math. (2) 100 (1974), 1-79.

18. A. Zabrodsky, On the realization of invariant subgroups of $\pi_{*}(X)$, revised preprint.

19. O. Zariski and P. Samucl, Commutative algebra, vol. 1, Van Nostrand, Princeton, N. J., 1960.

Mathematisches Institut, Bunsenstrasse 3/5, D-3400 Gottingen, Federal Republic of (jERMANY

Institut des Hautes Scientifiques, 35, route de Chartres, 91140 Bures-sur-Yvette, Les Vlis, FRANCE 